\title{
Capacity Bounds for Relay-Aided Wireless Multiple Multicast with Backhaul
}

\author{
Jinfeng Du, Ming Xiao, and Mikael Skoglund \\ ACCESS Linnaeus Center, Royal Institute of Technology, Stockholm, Sweden \\ Email: jinfeng@kth.se, ming.xiao@ee.kth.se, mikael.skoglund@ee.kth.se
}

\begin{abstract}
We investigate the capacity bounds for relay-aided two-source two-destination wireless networks with backhaul support between source nodes. Each source multicasts its own message to all destinations with the help of an intermediate relay node, which is full-duplex and shared by both sources. We are aiming to characterize the capacity region of this model given discrete memoryless Gaussian channels. We establish three capacity upper bounds by relaxing the cut-set bound, and by extending two capacity bounds originally derived for MIMO relay channels. We also present one lower bound by using decodingand-forward relaying combined with network beam-forming.
\end{abstract}

\section{INTRODUCTION}

Wireless communications have recently seen rapid progress both in academy and industry, and the use of relay as well as advanced cooperative communication techniques has the potential to further boost both the communication range and data rate. The full understanding of such systems, even for the original three-node relay network, is still not ready yet. In the last 30 years, numerous research efforts have been casted on the relay networks. In [1], [2], capacity bounds and various cooperative strategies for three-node relaying networks (sourcerelay-sink, or two cooperative sources and one sink) have been studied, with successive decoding, sliding-window forward decoding, or backward decoding techniques used at the sink. The relay (or the other source) uses decode-and-forward (DF) or compress-and-forward (CF) to aid the transmission. In [3] cooperative strategies and coding schemes are investigated for multiple-access relay channels (MARC) involving multiple sources and a single destination, and for broadcast relay channels (BRC) where a single source transmits messages to multiple destinations. Recent results on capacity bounds for multiple-source multiple-destination relay networks, [4][6] and references therein, have provided valuable insights into the benefits of relaying, either half-duplex or full-duplex. Apart from introducing dedicated relay nodes to help the transmission, one can also utilize cooperative strategies among sources and/or among destinations [7], [8] with the help of orthogonal conferencing channels.

In this paper, we aim to characterize the capacity regions when source cooperation and relaying are combined together. More specifically, we focus on a relay-aided two-source twodestination multicast network with backhaul support, as shown in Figure. 1. Source $\mathcal{S}_{1}$ intends to multicast ${ }^{1}$ its message $W_{1}$

\footnotetext{
${ }^{1}$ In some other papers and books, "multicast" is also referred as "broadcast" but with only common messages.
}

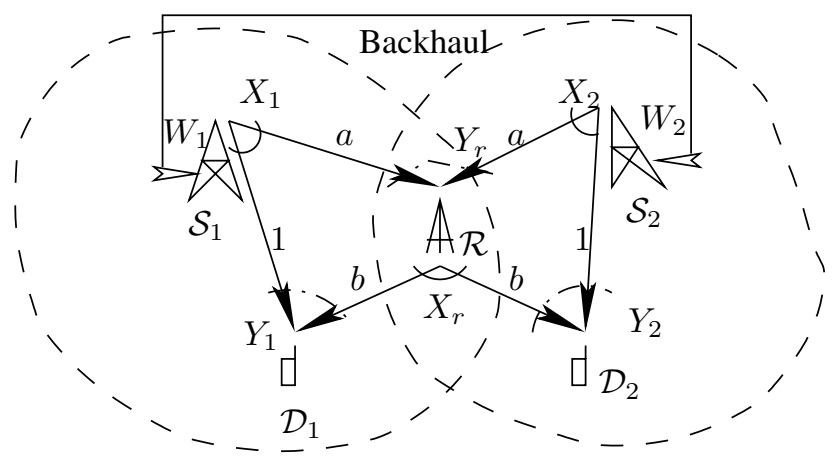

Figure 1. Two source nodes $\mathcal{S}_{1}$ and $\mathcal{S}_{2}$, connected with backhaul, multicast information $W_{1}$ and $W_{2}$ respectively to both destinations $\mathcal{D}_{1}$ and $\mathcal{D}_{2}$, with aid from a full-duplex relay node $\mathcal{R}$.

at rate $R_{1}$ to two geographically separated destinations $\mathcal{D}_{1}$ and $\mathcal{D}_{2}$, with the help of a relay $\mathcal{R}$. At the same time, source $\mathcal{S}_{2}$ also multicasts its message $W_{2}$ at rate $R_{2}$ to both destinations. The relay will forward the information it receives in previous time slot to both destinations. The transmissions from two sources and from the relay use the same channel resource (i.e. co-channel transmission) and will mix up at all the receiving terminals $\left(\mathcal{S}_{1}, \mathcal{S}_{2}\right.$, and $\left.\mathcal{R}\right)$. This model arises from downlink wireless cellular networks where two base stations multicast to two mobile terminals, one in each cell, with the help of a dedicated relay deployed at the common cell boundary. This model is interesting since it is a combination of relaying, MARC, BRC, and sources cooperation. It can be extended to more general networks by tuning the channel gains within the range $[0, \infty)$. In this paper, we are interested in the scenario without cross channels between $\mathcal{S}_{1}$ and $\mathcal{D}_{2}$, or $\mathcal{S}_{2}$ and $\mathcal{D}_{1}$. In wireless cellular networks, such cross channels are normally too weak to be used or technically suppressed by the system.

The rest of this paper is organized as follows. The system model is introduced in Section II. A lower bound given by networked beam-forming is presented in Section III, and three capacity upper bounds are established in Section IV. Numerical results are presented in Section V and concluding remarks are shown in Section VI.

Notations: Capital letter $X$ indicates a real valued random variable and $p(X)$ indicates its probability density/mass function. $X^{(n)}$ denotes a vector of random variables of length $n$ and $I(X ; Y)$ denotes the mutual information between $X$ and $Y$. $C(x)=\frac{1}{2} \log _{2}(1+x)$ is the Gaussian capacity function. 


\section{System ModeL}

To simplify our analysis, we consider a symmetric channel gain scenario in Figure 1 (extension to non-symmetric channel gains is straightforward),

$$
\begin{aligned}
& Y_{1}^{(n)}=X_{1}^{(n)}+b X_{r}^{(n)}+Z_{1}^{(n)}, \\
& Y_{2}^{(n)}=X_{2}^{(n)}+b X_{r}^{(n)}+Z_{2}^{(n)}, \\
& Y_{r}^{(n)}=a X_{1}^{(n)}+a X_{2}^{(n)}+Z_{r}^{(n)},
\end{aligned}
$$

where $a \geq 0$ is the normalized channel gain for the sourcerelay links and $b \geq 0$ for the relay-destination links. $X_{i}^{(n)}$, $Y_{i}^{(n)}, Z_{i}^{(n)}, i=1,2, r$ are $n$-dimensional transmitted signals, received signals, and noise, respectively. The noise components $Z_{i}(k), i=1,2, r$ and $k=1, \ldots, n$ are i.i.d. zero-mean unit-variance Gaussian random variables. Assuming perfect synchronization, $\mathcal{S}_{1}$ and $\mathcal{S}_{2}$ can cooperate with $\mathcal{R}$ and get coherent combining gains (i.e., beamforming) at the sinks, as stated in [1]-[3]. An average power constraint

$$
\frac{1}{n} \sum_{k=1}^{n} X_{i}^{2}(k) \leq P_{i}, \quad i=1,2, r,
$$

is assumed throughout this paper. We note that in practice, the backhaul has much higher capacity and lower error rates than the forward wireless channels. Therefore, in our model the backhaul is assumed to be error-free and of sufficiently high capacity, which makes our system closely related to the MIMO relay channel scenario, as studied in [9], [10]. However, we emphasize three main differences between the system investigated in this paper and the MIMO relay scenario with a two-antenna source node. Firstly, in our system each source/antenna is subject to an individual power constraint as stated in 2, while in the MIMO relay channel model a sum-power constraint is applied at the source node, which essentially means a larger achievable rate region. Secondly, in our system the relay combines messages from each source by performing $\mathrm{NC}$ rather than forwarding them separately through orthogonal channels. Since network coding is preferred in symmetric rate scenarios (otherwise we have to append zeros at the shorter message), its efficiency is limited by the sourcerelay channels, especially when the two source-relay channels are not symmetry. Last but no the least, our system model can be easily extended to the finite-rate backhaul scenario where only partial cooperation between source nodes is possible. Therefore, capacity lower bounds derived for the MIMO relay channel may not be directly relevant to our scenario. However, the corresponding upper bounds, e.g., Theorem 3.1 in [9] and the upper bound (9) in [10], are still valid. We will introduce them to our system and modify them accordingly to establish new upper bounds.

\section{CAPACITy LOWER BOUND}

A cooperative transmission strategy, namely networked beam-forming (NBF) with a full duplex decode-and-forward relay, has been proposed in [13] for non-perfectly synchronized signal at the relay. We will introduce the achievable rate presented in [13] but for perfectly synchronization scenario to serve as the capacity lower bound. The main results of NBF are listed here with a brief outline of the constructive proof.

Similar to [1]-[4], source $\mathcal{S}_{i}, i=1,2$, divides its messages $W_{i}$ into $B$ blocks $W_{i, 1}, \ldots, W_{i, B}$ with $n R_{i}$ bits each. The signals transmitted at $\mathcal{S}_{1}, \mathcal{S}_{2}$ and $\mathcal{R}$ are formulated in a beamforming fashion to take advantage of the coherent combining gain. The transmission process requires $B+2$ blocks in total, and each transmission is over $n$ channel uses, and assuming the backhual is used for free, the overall rate is $\frac{B k_{i}}{(B+2) n}$ bits per channel use, which converges to $R_{i}=\frac{k_{i}}{n}$ when $B$ goes to infinity.

During block $b-1,\left(W_{1, b-1}, W_{2, b-1}\right)$ are exchanged via the backhaul and formulated into a network coded message $W_{b}=f\left(W_{1, b-1}, W_{2, b-1}\right)$ by some function $f(\cdot)$; at block $b$ the coded message $W_{b}$ is transmitted by both sources and the relay receives and decodes it afterwards; at block $b+1, W_{b}$ is transmitted by $\mathcal{R}$, i.e.,

$$
X_{r, b+1}^{(n)}=\sqrt{P_{r}} U^{(n)}\left(W_{b}\right) .
$$

$U^{(n)}(W)$ is a codeword of the message $W$, and we relate their dependence in the way of an encoding function. Each codeword is generated in the usual memoryless fashion. Since the message $W_{b}$ (hence the signal $X_{r, b+1}^{(n)}$ ) at the relay is known by both sources before its transmission, $\mathcal{S}_{1}$ and $\mathcal{S}_{2}$ can cooperative their transmission of new message $W_{b+1}$ with the relaying message $W_{b}$ and transmit at block $b+1$

$$
X_{1, b+1}^{(n)}=\sqrt{\alpha_{1} P_{1}} V^{(n)}\left(W_{b+1}, W_{b}\right)+\sqrt{\left(1-\alpha_{1}\right) P_{1}} U^{(n)}\left(W_{b}\right),
$$$$
X_{2, b+1}^{(n)}=\sqrt{\alpha_{2} P_{2}} V^{(n)}\left(W_{b+1}, W_{b}\right)+\sqrt{\left(1-\alpha_{2}\right) P_{2}} U^{(n)}\left(W_{b}\right),
$$

where $0 \leq \alpha_{1}, \alpha_{2} \leq 1$ are power allocation parameters.

The decoding process is as follows: the relay performs successive decoding [1] to decode $W_{b}, b=1,2, \ldots, B$, and the destinations utilize backward decoding [11] to decode $W_{b}, b=B, B-1, \ldots, 1$. Since $\mathcal{S}_{1}$ and $\mathcal{S}_{2}$ transmit the same NC message $W_{b}$, the achievable sum-rate can be split arbitrarily between them. Therefore in NBF strategy only the constraints over the sum-rate matter. The following rate region is achievable by NBF,

$$
\begin{array}{r}
R_{1}+R_{2}<\min \left\{C\left(P_{1}+b^{2} P_{r}+2 b \sqrt{\left(1-\alpha_{1}\right) P_{1} P_{r}}\right),\right. \\
C\left(a^{2}\left(\sqrt{\alpha_{1} P_{1}}+\sqrt{\alpha_{2} P_{2}}\right)^{2}\right), \\
\left.C\left(P_{2}+b^{2} P_{r}+2 b \sqrt{\left(1-\alpha_{2}\right) P_{2} P_{r}}\right)\right\},
\end{array}
$$

with the union taken over the power allocation parameters $0 \leq \alpha_{1}, \alpha_{2} \leq 1$. The terms in (3) indicate the constraints at $\mathcal{D}_{1}, \mathcal{R}$, and $\mathcal{D}_{2}$, respectively.

For the symmetric scenario where $P_{1}=P_{2}=P_{r}=P$ (therefore $R_{1}=R_{2}=R$ ), by setting $\alpha_{1}=\alpha_{2}=\alpha$ in (3), the achievable symmetric rate $R$ is given by

$$
R<\max _{0 \leq \alpha \leq 1} \min \left\{\frac{1}{2} C\left(4 a^{2} P \alpha\right), \frac{1}{2} C\left(\left(1+b^{2}+2 b \sqrt{1-\alpha}\right) P\right)\right\} .
$$




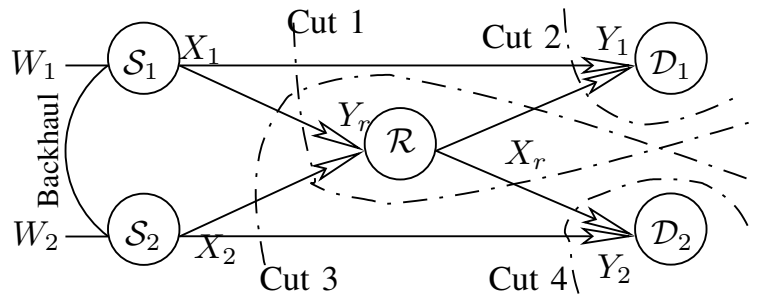

Figure 2. The sum multicast capacity is bounded by the cut-set bound based on the four cuts shown in the figure.

\section{CAPACITY UPPER BOUNDS}

The cut-set bound [12] on the sum rate $R_{1}+R_{2}$ will be derived for our model, and two upper bounds for the MIMO relay channels given by [9] and [10] will also be discussed as references.

\section{A. Upper Bound from the Cut-Set Bound}

As in [12], the cut-set bound for the sum-rate $R_{1}+R_{2}$ over the cooperative relay network shown in Fig. 1 can be derived based on the four cuts shown in Fig. 2, i.e.,

$$
\begin{aligned}
& C_{c u t-s e t}=\sup _{p\left(X_{1}, X_{2}, X_{r}\right)} \min \left\{I\left(X_{1}, X_{2} ; Y_{1}, Y_{r} \mid X_{r}\right),\right. \\
& \left.I\left(X_{1}, X_{r} ; Y_{1}\right), I\left(X_{1}, X_{2} ; Y_{2}, Y_{r} \mid X_{r}\right), I\left(X_{2}, X_{r} ; Y_{2}\right)\right\} .
\end{aligned}
$$

To model the potential correlation among $X_{1}, X_{2}$ and $X_{r}$ due to cooperation, we partition the transmitting signals as follows

$X_{r}^{(n)}=\sqrt{P_{r}} U^{(n)}$,

$X_{1}^{(n)}=\sqrt{\alpha_{1}^{\prime} P_{1}} S_{1}^{(n)}+\sqrt{\alpha_{1}^{\prime \prime} P_{1}} V^{(n)}+\sqrt{\left(1-\alpha_{1}^{\prime}-\alpha_{1}^{\prime \prime}\right) P_{1}} U^{(n)}$,

$X_{2}^{(n)}=\sqrt{\alpha_{2}^{\prime} P_{2}} S_{2}^{(n)}+\sqrt{\alpha_{2}^{\prime \prime} P_{2}} V^{(n)}+\sqrt{\left(1-\alpha_{2}^{\prime}-\alpha_{2}^{\prime \prime}\right) P_{2}} U^{(n)}$,

where $S_{1}, S_{2}, V$, and $U$ are independent random variables with zero-mean and unit-variance to represent respectively, the individual signals for user 1 and user 2, the cooperative signal between two sources, and the cooperative signal with the relay. The received signals in (1) therefore becomes

$$
\begin{gathered}
Y_{1}^{(n)}=\left(b \sqrt{P_{r}}+\sqrt{\left(1-\alpha_{1}^{\prime}-\alpha_{1}^{\prime \prime}\right) P_{1}}\right) U^{(n)}+\sqrt{\alpha_{1}^{\prime} P_{1}} S_{1}^{(n)} \\
+\sqrt{\alpha_{1}^{\prime \prime} P_{1}} V^{(n)}+Z_{1}^{(n)}, \\
Y_{2}^{(n)}=\left(b \sqrt{P_{r}}+\sqrt{\left(1-\alpha_{2}^{\prime}-\alpha_{2}^{\prime \prime}\right) P_{2}}\right) U^{(n)}+\sqrt{\alpha_{2}^{\prime} P_{2}} S_{2}^{(n)} \\
+\sqrt{\alpha_{2}^{\prime \prime} P_{2}} V^{(n)}+Z_{2}^{(n)}, \\
Y_{r}^{(n)}=a\left(\sqrt{\alpha_{1}^{\prime \prime} P_{1}}+\sqrt{\alpha_{2}^{\prime \prime} P_{2}}\right) V^{(n)}+a \sqrt{\alpha_{1}^{\prime} P_{1}} S_{1}^{(n)}+Z_{r}^{(n)} \\
+a \sqrt{\alpha_{2}^{\prime} P_{2}} S_{2}^{(n)}+a\left(\sqrt{\left(1-\alpha_{1}^{\prime}-\alpha_{1}^{\prime \prime}\right) P_{1}}+\sqrt{\left(1-\alpha_{2}^{\prime}-\alpha_{2}^{\prime \prime}\right) P_{2}}\right) U^{(n)} .
\end{gathered}
$$

For Cut 2 and Cut 4, it can be verified that [12]

$$
\begin{aligned}
& I\left(X_{1}, X_{r} ; Y_{1}\right) \leq C\left(P_{1}+b^{2} P_{r}+2 b \sqrt{\left(1-\alpha_{1}^{\prime}-\alpha_{1}^{\prime \prime}\right) P_{1} P_{r}}\right), \\
& I\left(X_{2}, X_{r} ; Y_{2}\right) \leq C\left(P_{2}+b^{2} P_{r}+2 b \sqrt{\left(1-\alpha_{2}^{\prime}-\alpha_{2}^{\prime \prime}\right) P_{2} P_{r}}\right),
\end{aligned}
$$

where the equalities are achieved by joint Gaussian distributed signals $\left(X_{1}, X_{2}, X_{r}\right)$. Note that for Cut 1 , we have

$$
\begin{gathered}
I\left(X_{1}, X_{2} ; Y_{1}, Y_{r} \mid X_{r}\right)=h\left(Y_{1}, Y_{r} \mid X_{r}\right)-h\left(Y_{1}, Y_{r} \mid X_{1}, X_{2}, X_{r}\right) \\
=h\left(Y_{r} \mid X_{r}, Y_{1}\right)+h\left(Y_{1} \mid X_{r}\right)-h\left(Y_{1} \mid X_{1}, X_{2}, X_{r}\right) \\
\quad-h\left(Y_{r} \mid X_{1}, X_{2}, X_{r}\right) \\
=h\left(Y_{r} \mid X_{r}, Y_{1}\right)+I\left(X_{1}, X_{2} ; Y_{1} \mid X_{r}\right)-h\left(Y_{r} \mid X_{1}, X_{2}, X_{r}\right) \\
=h\left(Y_{r} \mid X_{r}, Y_{1}\right)-h\left(Y_{r} \mid X_{r}\right)+I\left(X_{1}, X_{2} ; Y_{1} \mid X_{r}\right) \\
\quad+h\left(Y_{r} \mid X_{r}\right)-h\left(Y_{r} \mid X_{1}, X_{2}, X_{r}\right) \\
=I\left(X_{1}, X_{2} ; Y_{1} \mid X_{r}\right)+I\left(X_{1}, X_{2} ; Y_{r} \mid X_{r}\right)-I\left(Y_{1} ; Y_{r} \mid X_{r}\right) .
\end{gathered}
$$

Similarly for Cut 3 we have

$I\left(X_{1}, X_{2} ; Y_{2}, Y_{r} \mid X_{r}\right)$

$=I\left(X_{1}, X_{2} ; Y_{2} \mid X_{r}\right)+I\left(X_{1}, X_{2} ; Y_{r} \mid X_{r}\right)-I\left(Y_{2} ; Y_{r} \mid X_{r}\right)$.

It is hard to find a suitable distribution $p\left(X_{1}, X_{2}, X_{r}\right)$ that can maximize (6) and (7). Therefore the direct calculation of $C_{c u t-s e t}$ turns out to be a hard problem to solve. Alternatively, given the fact that $I\left(Y_{1} ; Y_{r} \mid X_{r}\right) \geq 0$ and $I\left(Y_{2} ; Y_{r} \mid X_{r}\right) \geq 0$, we can find an upper bound $C_{\text {upper } 1} \geq C_{\text {cut-set }}$ by removing the $I\left(Y_{1} ; Y_{r} \mid X_{r}\right)$ and $I\left(Y_{2} ; Y_{r} \mid X_{r}\right)$ from (6) and (7), respectively,

$I\left(X_{1}, X_{2} ; Y_{1}, Y_{r} \mid X_{r}\right) \leq I\left(X_{1}, X_{2} ; Y_{1} \mid X_{r}\right)+I\left(X_{1}, X_{2} ; Y_{r} \mid X_{r}\right)$, $I\left(X_{1}, X_{2} ; Y_{2}, Y_{r} \mid X_{r}\right) \leq I\left(X_{1}, X_{2} ; Y_{2} \mid X_{r}\right)+I\left(X_{1}, X_{2} ; Y_{r} \mid X_{r}\right)$.

Since all the items in RHS of (8) are simultaneously maximized by joint Gaussian distribution, i.e.,

$$
\begin{aligned}
& I\left(X_{1}, X_{2} ; Y_{1} \mid X_{r}\right) \leq C\left(\left(\alpha_{1}^{\prime}+\alpha_{1}^{\prime \prime}\right) P_{1}\right), \\
& I\left(X_{1}, X_{2} ; Y_{2} \mid X_{r}\right) \leq C\left(\left(\alpha_{2}^{\prime}+\alpha_{2}^{\prime \prime}\right) P_{2}\right), \\
& I\left(X_{1}, X_{2} ; Y_{r} \mid X_{r}\right) \leq \\
& C\left(a^{2}\left[\left(\alpha_{1}^{\prime}+\alpha_{1}^{\prime \prime}\right) P_{1}+\left(\alpha_{2}^{\prime}+\alpha_{2}^{\prime \prime}\right) P_{2}+2 \sqrt{\alpha_{1}^{\prime \prime} \alpha_{2}^{\prime \prime} P_{1} P_{2}}\right]\right),
\end{aligned}
$$

by combining it with (5), we can find the upper bound as

$$
\begin{aligned}
& R_{1}+R_{2}<C_{\text {upper } 1}=\underset{\substack{\alpha_{1}^{\prime}, \alpha_{1}^{\prime \prime}, \alpha_{2}^{\prime}, \alpha_{2}^{\prime \prime} \geq 0 \\
0 \leq \alpha_{1}^{\prime}+\alpha_{1}^{\prime \prime} \leq 1,0 \leq \alpha_{2}^{\prime}+\alpha_{2}^{\prime \prime} \leq 1}}{\min } \\
& \left\{C\left(P_{1}+b^{2} P_{r}+2 b \sqrt{\left(1-\alpha_{1}^{\prime}-\alpha_{1}^{\prime \prime}\right) P_{1} P_{r}}\right),\right. \\
& C\left(P_{2}+b^{2} P_{r}+2 b \sqrt{\left(1-\alpha_{2}^{\prime}-\alpha_{2}^{\prime \prime}\right) P_{2} P_{r}}\right), \\
& C\left(\left(\alpha_{1}^{\prime}+\alpha_{1}^{\prime \prime}\right) P_{1}\right)+ \\
& C\left(a^{2}\left[\left(\alpha_{1}^{\prime}+\alpha_{1}^{\prime \prime}\right) P_{1}+\left(\alpha_{2}^{\prime}+\alpha_{2}^{\prime \prime}\right) P_{2}+2 \sqrt{\alpha_{1}^{\prime \prime} \alpha_{2}^{\prime \prime} P_{1} P_{2}}\right]\right), \\
& C\left(\left(\alpha_{2}^{\prime}+\alpha_{2}^{\prime \prime}\right) P_{2}\right)+ \\
& \left.C\left(a^{2}\left[\left(\alpha_{1}^{\prime}+\alpha_{1}^{\prime \prime}\right) P_{1}+\left(\alpha_{2}^{\prime}+\alpha_{2}^{\prime \prime}\right) P_{2}+2 \sqrt{\alpha_{1}^{\prime \prime} \alpha_{2}^{\prime \prime} P_{1} P_{2}}\right]\right)\right\} .
\end{aligned}
$$

For the symmetric rate scenario where $P_{1}=P_{2}=P_{r}=P$, by setting $\alpha_{1}^{\prime}=\alpha_{2}^{\prime}=\alpha^{\prime}$ and $\alpha_{1}^{\prime \prime}=\alpha_{2}^{\prime \prime}=\alpha^{\prime \prime}$ (10) becomes

$$
\begin{gathered}
C_{\text {upper } 1}^{R}=\sup _{\substack{\alpha^{\prime}, \alpha^{\prime \prime} \geq 0 \\
0 \leq \alpha^{\prime}+\alpha^{\prime \prime} \leq 1}} \min \left\{\frac { 1 } { 2 } C \left(P\left(1+b^{2}+2 b \sqrt{1-\alpha^{\prime}-\alpha^{\prime \prime}}\right),\right.\right. \\
\left.1 / 2 *\left[C\left(\left(\alpha^{\prime}+\alpha^{\prime \prime}\right) P\right)+C\left(2 a^{2} P\left(\alpha^{\prime}+2 \alpha^{\prime \prime}\right)\right)\right]\right\} .
\end{gathered}
$$




\section{B. Upper Bounds from MIMO Relay Channels}

As stated in Sec. II, by modifying the channel and power allocation parameters accordingly (real signal and noise, single receiver antenna), the capacity upper bounds given by [9] and [10] are still valid and therefore can serve as baselines to bound the capacity regions.

As in Theorem 3.1 of [9], we can group the two singleantenna source nodes together for a new source node with $M_{1}=2$ transmit antennas. The relay has $N_{1}=1$ receive antenna and $M_{2}=1$ transmit antenna. Each of the destination node has $N=1$ receive antenna. Since the transmitting power constraint has been incorporated into the signals $X_{i}$ as described in (2), we simply set the power parameters $\eta_{1}=\eta_{2}=\eta_{3}=1$. According to the system model described in (1a) and (1c), the virtual MIMO relay channel defined by $\mathcal{S}_{1}, \mathcal{S}_{2}, \mathcal{R}$ and $\mathcal{D}_{1}$ has the following channel matrices: The source-relay channel $H_{1}=[a, a]$, the source-destination channel $H_{2}=[1,0]$ and the relay-destination channel $H_{3}=b$. We can set the covariance of $X_{r}$ as $\Sigma_{22}=E\left[X_{r}^{2}\right]=P_{r}$ and the covariance matrix of $\left[\begin{array}{ll}X_{1} & X_{2}\end{array}\right]$ as

$$
\Sigma_{11}=E\left\{\left[\begin{array}{ll}
X_{1} & X_{2}
\end{array}\right]^{\prime} *\left[\begin{array}{ll}
X_{1} & X_{2}
\end{array}\right]\right\}=\left[\begin{array}{cc}
P_{1} & \lambda \sqrt{P_{1} P_{2}} \\
\lambda \sqrt{P_{1} P_{2}} & P_{2}
\end{array}\right],
$$

where $0 \leq \lambda \leq 1$ is introduced to model the potential correlation between $X_{1}$ and $X_{2}$. The capacity upper bound defined by Theorem 3.1 of [9] therefore can be written as

$$
R_{1}+R_{2}<C_{\text {upper } 2}=\max _{0 \leq \rho, \lambda \leq 1} \min \left\{C_{1}^{G}, C_{2}^{G}, C_{3}^{G}, C_{4}^{G}\right\},
$$

where $C_{1}^{G}$ and $C_{2}^{G}$ are obtained from (8) and (9) in [9] and $C_{3}^{G}$ and $C_{4}^{G}$ by replacing $H_{2}$ by $\hat{H}_{2}=[0,1]$.

For symmetric scenarios, we can translate (12) to bound the symmetric rate $R_{1}=R_{2}=R$ as follows

$$
\begin{gathered}
R<C_{\text {upper } 2}^{R}=\max _{0 \leq \rho, \lambda \leq 1} \min \left\{\frac{1}{2} C\left(P\left(1+b^{2}+2 b \rho\right)\right),\right. \\
\left.\frac{1}{2} C\left(P\left(1-\rho^{2}\right)\left[1+2 a^{2}(1+\lambda)+a^{2} P\left(1-\rho^{2}\right)\left(1-\lambda^{2}\right)\right]\right)\right\} .
\end{gathered}
$$

Note that the bound $C_{\text {upper } 2}$ is not tight in general for two reasons: (i) the equality in (4) of [9] is achieved only if $M_{1} \leq M_{2}$, which is not the case here; (ii) the optimization (12) is non-convex on $\rho$ and therefore cannot guarantee global optimum. The overcome these limitations, a joint covariance matrix has been introduced in [10] as follows

$$
\boldsymbol{R}_{S R}=\left[\begin{array}{ccc}
P_{1} & \lambda \sqrt{P_{1} P_{2}} & \rho \sqrt{P_{1} P_{r}} \\
\lambda \sqrt{P_{1} P_{2}} & P_{2} & \mu \sqrt{P_{2} P_{r}} \\
\rho \sqrt{P_{1} P_{r}} & \mu \sqrt{P_{2} P_{r}} & P_{r}
\end{array}\right],
$$

where $0 \leq \rho, \lambda, \mu \leq 1$ are correlation coefficients. By setting the number of antennas $N_{s}=2$ at the source, $N_{R}=1$ at Relay and $N_{D}=1$ at destinations, with channel matrices

$$
H_{0}=[1,0], H_{1}=[a, a], H_{2}=b, \hat{H}_{0}=[0,1]
$$

and the auxiliary construction matrices

$$
\boldsymbol{D}_{S}=\left[\begin{array}{lll}
1 & 0 & 0 \\
0 & 1 & 0
\end{array}\right], \boldsymbol{D}_{R}=\left[\begin{array}{lll}
0 & 0 & 1
\end{array}\right]
$$

the upper bound given by (9) of [10] can be written as

$$
\begin{array}{r}
R_{1}+R_{2}<C_{\text {upper } 3}=\max _{0 \leq \rho, \lambda, \mu \leq 1} \min \\
\left\{C\left(P_{1}+b^{2} P_{r}+2 b \rho \sqrt{P_{1} P_{r}}\right),\right. \\
C\left(P_{2}+b^{2} P_{r}+2 b \mu \sqrt{P_{2} P_{r}}\right), \\
\frac{1}{2} \log _{2}\left(\left(1+P_{1}\right)\left(1+a^{2}\left(P_{1}+P_{2}+2 \lambda \sqrt{P_{1} P_{2}}\right)\right)\right. \\
\left.-a^{2}\left(P_{1}+\lambda \sqrt{P_{1} P_{2}}\right)^{2}\right), \\
\frac{1}{2} \log _{2}\left(\left(1+P_{2}\right)\left(1+a^{2}\left(P_{1}+P_{2}+2 \lambda \sqrt{P_{1} P_{2}}\right)\right)\right. \\
\left.\left.-a^{2}\left(P_{2}+\lambda \sqrt{P_{1} P_{2}}\right)^{2}\right)\right\} .
\end{array}
$$

For symmetric scenarios, we can get from (14) that

$$
\begin{array}{r}
R<C_{\text {upper } 3}^{R}=\max _{0 \leq \rho \leq \lambda \leq 1} \min \left\{\frac{1}{2} C\left(P\left(1+b^{2}+2 b \rho\right)\right),\right. \\
\left.\frac{1}{2} C\left(P\left(1+2 a^{2}(1+\lambda)+a^{2} P\left(1-\lambda^{2}\right)\right)\right)\right\} .
\end{array}
$$

Note that the upper bound given by (9) of [10] is derived based on the sum-power constraint, which means it is in general loose for our case where only per-antenna/user power constraint is applied.

\section{A Tighter Upper Bound $C_{\text {upp }}$}

Based on the upper bounds $C_{\text {upper } 1}, C_{\text {upper } 2}$ and $C_{\text {upper } 3}$, we can obtain a tighter upper bound by taking their minimum,

$$
R_{1}+R_{2}<C_{\text {upp }}=\min \left\{C_{\text {upper } 1}, C_{\text {upper } 2}, C_{\text {upper } 3}\right\} .
$$

It is similar for the symmetric rate upper bound $C_{u p p}^{R}$.

\section{NUMERICAL RESUlTS}

In this section, we present the numerical results on the capacity bounds on the symmetric rate scenarios with different power and channel gain parameters to compare these bound for different link quality. For power constraint $P_{1}=P_{2}=P_{r}=P$ and channel gains $a^{2}$ and $b^{2}$, the Signal-to-noise ratios are $P / \sigma^{2}$ for the source-destination link, $a^{2} P / \sigma^{2}$ for the sourcerelay link, and $b^{2} P / \sigma^{2}$ for the relay-destination link, respectively. The results for non-symmetric cases are similar and therefore omitted.

In Fig. 3, we compare these upper bounds discussed in Section IV with fixed transmitting power $P / \sigma^{2}=5 \mathrm{~dB}$ and relay-destination channel gain $b^{2}=0 \mathrm{~dB}$ but varying the source-relay channel gain $a^{2}$. When the source-relay channel is weak, the scenario where the DF relay strategy performs bad, the gap between upper and lower bounds is large, upp to 0.5 bits per channel use. When $a^{2}$ is large, however, the NBF scheme together with a DF relay turns to be optimal.

In Fig. 4, we keep transmitting power $P / \sigma^{2}=5 \mathrm{~dB}$ and the relay-destination channel gain $a^{2}=0 \mathrm{~dB}$ and but varying relay-destination channel gain $b^{2}$. For a weak relay-destination channel, NBF with DF relay performs well and the capacity gap is small, within 0.03 bits per channel use. For strong relaydestination channel, the gap is 0.16 bits per channel use. 


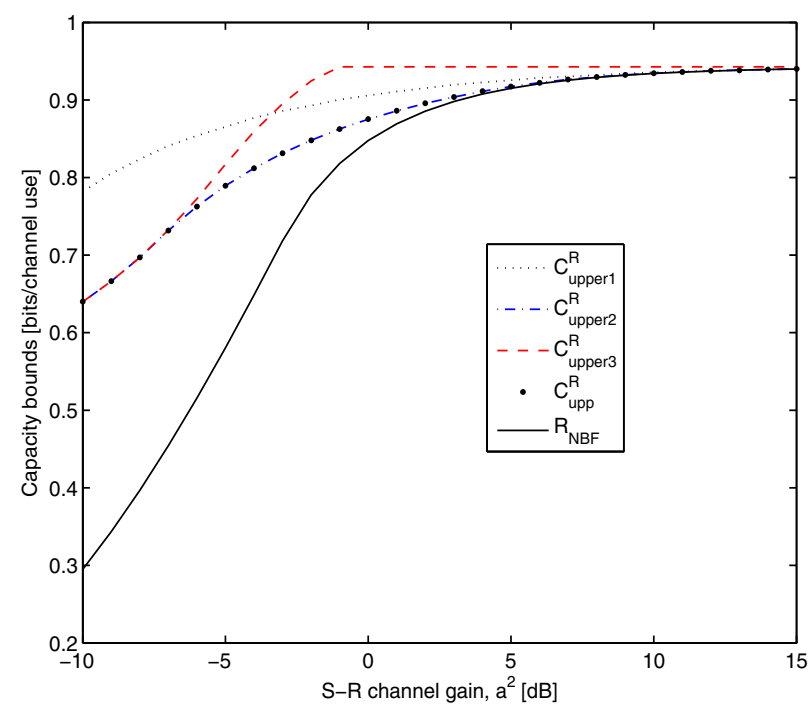

Figure 3. Capacity bounds for varying source-relay channel gain $a^{2}$ with fixed transmitting power $P / \sigma^{2}=5 \mathrm{~dB}$ and relay-destination channel gain $b^{2}=0 d B$.

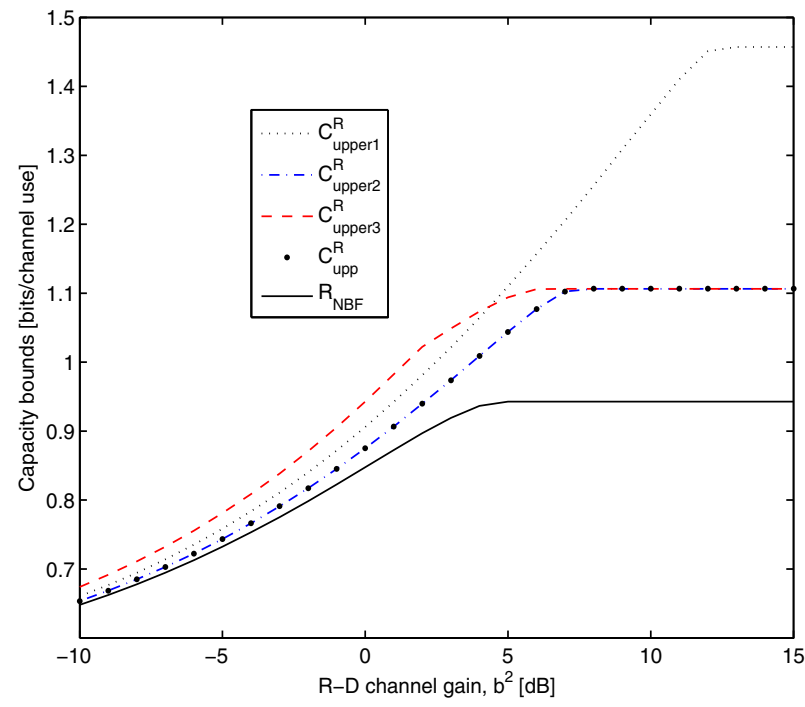

Figure 4. Capacity bounds for varying relay-destination channel gain $b^{2}$ with fixed transmitting power $P / \sigma^{2}=5 \mathrm{~dB}$ and source-relay channel gain $a^{2}=0 d B$.

In Fig. 5, we investigate the asymptotic performance of different bounds with varying transmitting power $P / \sigma^{2}$ but fixed source-relay channel gain $a^{2}=0 \mathrm{~dB}$ and relay-destination channel gain $b^{2}=0 \mathrm{~dB}$. A capacity gap of 0.07 bits per channel use can be observed at high SNR.

\section{CONCLUSIONS}

We have studied a relay-aided two-source two-sink wireless multicast network with a backhual link between the source nodes. We provided three upper bounds on the capacity region and one lower bound given by an cooperative strategies using a full-duplex DF relay. The gap between the upper bounds and the lower bounds are still large in most of the regions

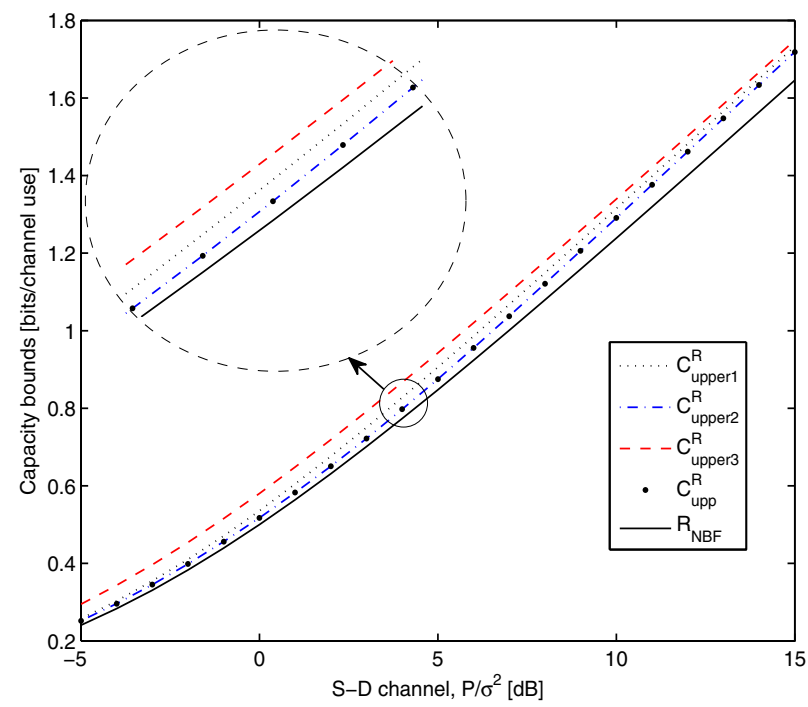

Figure 5. Capacity bounds for varying transmitting power $P / \sigma^{2}$ but fixed source-relay channel gain $a^{2}=0 \mathrm{~dB}$ and relay-destination channel gain $b^{2}=$ $0 d B$.

but converge in some specific cases, as illustrated in the our numerical results. Further research on the capacity bounds are needed to make deeper understanding of the capacity regions of such building blocks in wireless networks.

\section{ACKNOWLEDGMENTS}

This work is funded by VINNOVA and Wireless@KTH.

\section{REFERENCES}

[1] T. M. Cover and A. El Gamal, "Capacity theorems for the relay channel," IEEE Trans. Inf. Theory, vol. 25, pp. 572-584, Sep. 1979.

[2] A. Høst-Madsen and J. Zhang, "Capacity bounds and power allocation for wireless relay channels," IEEE Trans. Inf. Theory, vol. 51, pp. 2020 2040, Jun. 2005.

[3] G. Kramer, M. Gastpar and P. Gupta, "Cooperative strategies and capacity theorems for relay networks," IEEE Trans. Inf. Theory, vol. 51, pp. 30373063, Sep. 2005.

[4] O. Sahin and E. Erkip, "Achievable rates for the Gaussian interference relay channel", in Proc. of IEEE GLOBECOM, Nov. 2007.

[5] S. Katti, I. Marić, A. J. Goldsmith, D. Katabi, and M. Médard, "Joint relaying and network coding in wireless networks", in Proc. of IEEE ISIT, Jun. 2007.

[6] D. Gündüz, O. Simeone, A. J. Goldsmith, H. V. Poor, and S. Shamai, "Multiple multicasts with the help of a relay," http://arxiv.org/abs/0902.3178v1

[7] C. T. K Ng, N. Jindal, A. J. Goldsmith, and U. Mitra, "Capacity gain from two-transmitter and two-receiver cooperation", IEEE Trans. Inf. Theory, vol. 53, pp. 3822-3827, Oct. 2007.

[8] O. Simeone, D. Gündüz, H. V. Poor, A. J. Goldsmith, and S. Shamai, "Compound multiple-access channels with partial cooperation," IEEE Trans. Inf. Theory, vol. 55, pp. 2425-2441, Jun. 2009.

[9] B. Wang, J. Zhang, and A. Høst-Madsen, "On the capacity of MIMO relay channels," IEEE Trans. Inf. Theory, vol. 51, pp. 29-43, Jan. 2005.

[10] S. Simoens, O. Muñoz-Medina, J. Vidal, and A. del Coso, "On the Gaussian MIMO relay channel with full channel state information," IEEE Trans. Signal Proc., vol. 57, pp. 3588-3599, Sep. 2009.

[11] A. b. Carleial, "Multiple-access channels with different generalized feedback signals," IEEE Trans. Inf. Theory, vol. 28, pp. 841-850, Nov. 1982.

[12] T. M. Cover and J. A. Thomas, Elements of Information Theory, New York, Wiley, 2006.

[13] J. Du, M. Xiao, and M. Skoglund, "Cooperative strategies for relay-aided multi-cell wireless networks with Backhaul," in Proc. of IEEE ITW, Aug. 2010. 